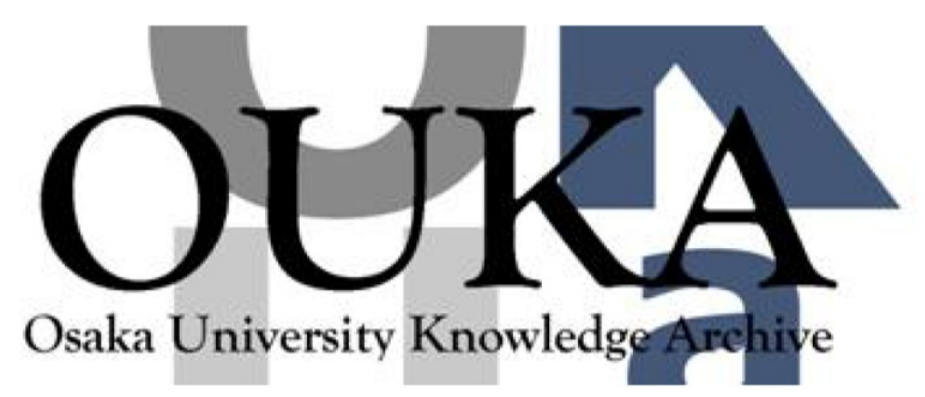

\begin{tabular}{|c|c|}
\hline Title & $\begin{array}{l}\text { Spin Diffusion in a Two-Dimensional Degenerate } \\
\text { Fermi Liquid }\end{array}$ \\
\hline Author (s) & Miyake, K.; Mullin, W. J. \\
\hline Citation & Physical Review Letters. 50(3) p. 197-p. 200 \\
\hline Issue Date & $1983-01-17$ \\
\hline oaire:version & VoR \\
\hline URL & https://hdl. handle. net/11094/3445 \\
\hline rights & $\begin{array}{l}\text { Miyake, K., Mullin, W. J., Physical Review } \\
\text { Letters, 50, 3, 197-200, 1983-01-17. "Copyright } \\
1983 \text { by the American Physical Society." }\end{array}$ \\
\hline Note & \\
\hline
\end{tabular}

Osaka University Knowledge Archive : OUKA

https://ir. Library. osaka-u. ac. jp/

Osaka University 


\title{
Spin Diffusion in a Two-Dimensional Degenerate Fermi Liquid
}

\author{
K. Miyake (a) and W. J. Mullin( ${ }^{(b)}$ \\ School of Mathematical and Physical Sciences, University of Sussex, Falmer, Brighton, Sussex BN1 9QH, England
} (Received 2 August 1982)

\begin{abstract}
The spin-diffusion coefficient $D$ of a polarized two-dimensional degenerate Fermi fluid is evaluated as a model of adsorbed ${ }^{3} \mathrm{He}$. Because in two dimensions, for zero or weak polarization, the only allowed scattering angles are 0 and $\pi$, the transport coefficients become expressible in terms of Landau parameters without approximation. For unpolarized and weakly polarized systems it is shown that $D^{-1} \sim T^{2} \ln T$ and $T^{2} \ln$ (polarization), respectively. The two-dimensional kinetic equation is found to be exactly solvable.
\end{abstract}

PACS numbers: $67.50 . \mathrm{Dg} .05 .60 .+\mathrm{w}, 66.90 .+\mathrm{r}, 67.70 .+\mathrm{n}$

Of the possible transport properties of submonolayer ${ }^{3} \mathrm{He}$ adsorbed on a substrate ${ }^{1}$ the one seemingly most accessible to experiment is spin diffusion, measured by NMR. For ${ }^{3} \mathrm{He}$ on Grafoil the time for a particle to diffuse to the edge of a substrate platelet is less than the relaxation time $T_{2}$, which invalidates the conditions necessary for a direct measurement of the spin-diffusion coefficient $D$ by use of spin-echo techniques. An indirect measurement of $D$ through $T_{2}$ measurements has been carried out ${ }^{2}$ in the fluid phase of adsorbed ${ }^{3} \mathrm{He}$, where relaxation is via field irregularities due to the substrate (analogous to the "bounded diffusion" technique ${ }^{3}$ ). These measurements were made at temperatures for which the fluid behaves as a classical gas (where $T_{2} \sim D$ $\sim \sqrt{T})$. Temperatures sufficiently low for adsorbed ${ }^{3} \mathrm{He}$ to behave as a degenerate two-dimensional (2D) Fermi fluid have barely, if at all, been reached. ${ }^{1,2}$ A possible recent exception to this may be the results of Bhattacharyya and Gasparini ${ }^{4}$ who claim to have found a degenerate liquid phase for ${ }^{3} \mathrm{He}$ on thin ${ }^{4} \mathrm{He}$ films. It is hoped that this Letter will encourage further experimental exploration of this regime which we show could be quite interesting. We examine spin diffusion $^{5}$ in a $2 \mathrm{D}$ Fermi fluid; we find the following results:

(1) The temperature dependence ${ }^{6}$ of $D$ is, for an unpolarized Fermi gas, $D^{-1} \sim T^{2} \ln \left(T_{f} / T\right)\left(T_{f}\right.$ $=$ Fermi temperature) in contrast to the $3 \mathrm{D}$ case for which $D^{-1} \sim T^{2}$. For a weakly polarized gas $\left(k T \ll \epsilon_{+}-\epsilon_{-} \ll \epsilon_{ \pm}\right)$the $\ln T$ behavior changes to $\ln P$, where $\epsilon_{\sigma}$ is the Fermi energy for spin $\sigma$ and $P$ is the polarization. For a highly polarized system the logarithm disappears and $D^{-1} \sim T^{2}$.

(2) It is found that an exact solution of the Boltzmann equation is possible as in the 3D Fermi gas.

(3) Because, as shown below, the allowed scattering angle in a 2D degenerate Fermi system has only two values, forward and backward, a Fermi-liquid theory description of both static ${ }^{7}$ and kinetic properties in terms of Landau parameters is more direct and unambiguous than in $3 \mathrm{D} .^{8}$

The kinetic equation will now be solved for arbitrary polarization. For the purposes of clear presentation the derivation will ignore Fermiliquid effects and the equation will be solved only in variational approximation. However, at the end we will quote the exact results, including Fermi-liquid effects, for the unpolarized case.

The kinetic equation is linearized as usual ${ }^{9}$ by adding - $\left(\partial n_{p \sigma} / \partial \epsilon_{p \sigma}\right) \Phi_{p \sigma}$ to the local equilibrium distribution function

$$
n_{p \sigma}=\left[\exp \left(x_{p \sigma}\right)+1\right]^{-1}
$$

with

$$
x_{p \sigma}=\beta\left(\epsilon_{p \sigma}-\hbar \gamma B-\mu_{\sigma}\right),
$$

where $\mu_{\sigma}$ is the chemical potential for spin $\sigma$, and $\beta=1 / k T$. The gradient in the magnetization which drives the spin current is assumed to be caused by gradients in the $\mu_{\sigma}$. The linearized Boltzmann equation becomes

$$
\begin{aligned}
\left(\partial n_{p \sigma} / \partial \epsilon_{p \sigma}\right) \overrightarrow{\mathrm{v}}_{p \sigma} \cdot \nabla \mu_{\sigma}=\left(\beta / A^{2}\right) \sum_{2,3,4} \delta \overrightarrow{\mathrm{p}}_{1}+\overrightarrow{\mathrm{p}}_{2}, \overrightarrow{\mathrm{p}}_{3}+\overrightarrow{\mathrm{p}}_{4} \delta\left(\epsilon_{1}+\epsilon_{2}-\epsilon_{3}-\epsilon_{4}\right) & \\
\quad & \quad\left\{\frac{1}{2} w_{\sigma \sigma} n_{1 \sigma} n_{2 \sigma}\left(1-n_{3 \sigma}\right)\left(1-n_{4 \sigma}\right)\left[\Phi_{1 \sigma}+\Phi_{2 \sigma}-\Phi_{3 \sigma}-\Phi_{4 \sigma}\right]\right. \\
& \left.\quad+w_{\sigma-\sigma} n_{1 \sigma} n_{2-\sigma}\left(1-n_{3 \sigma}\right)\left(1-n_{4-\sigma}\right)\left[\Phi_{1 \sigma}+\Phi_{2-\sigma}-\Phi_{3 \sigma}-\Phi_{4-\sigma}\right]\right\} .
\end{aligned}
$$

Particles 1 and 2 are incoming, 3 and 4 outgoing; $w_{\sigma \sigma^{\prime}}$ is the scattering probability for collision be- 
tween spins $\sigma$ and $\sigma^{\prime} . A$ is the sample area. The appropriate form for $\Phi_{p \sigma}$ is

$$
\Phi_{p_{i} \sigma}=-v_{p_{i} \sigma}\left|\nabla \mu_{\sigma}\right| q_{\sigma} \cos \Theta_{i}
$$

in which $q_{0}$, which should depend on $x_{i}$, is taken as a constant in our variational solution and $\Theta_{i}$ is the angle between $\overrightarrow{\mathrm{p}}_{i}$ and $\nabla \mu_{\sigma}$. The spin current for spin $\sigma$ is

$$
\vec{J}_{\sigma}=\sum_{p \sigma} n_{p \sigma} \vec{\nabla}_{p \sigma}=-N(0) p_{\sigma}^{2} q_{\sigma} \nabla \mu_{\sigma} / 4 m^{2}
$$

where $N(0)=m / \pi \hbar^{2}$ is the density of states and $p_{\sigma}$ is the Fermi momentum for spin $\sigma$. By use of the Gibbs-Duhem relation at constant temperature and pressure, one can relate $\nabla \mu_{\sigma}$ to $\nabla\left(n_{+}\right.$ $-n_{-}$) where $n_{\sigma}$ is the spin- $\sigma$ particle density. Then because total particle current must vanish in a diffusion experiment we can show that $q_{+}$ $=q_{-} \equiv q$ and

$$
D=4 \pi \hbar^{2} n_{+} n_{-} q / m^{2} n
$$

where $n=n_{+}+n_{-}$.

The sum over $p_{4}$ in Eq. (3) is carried out using the momentum $\delta$ function and the sums over $\overrightarrow{\mathrm{p}}_{2}$, $\overrightarrow{\mathrm{p}}_{3}$ are converted to integrals over $\epsilon_{2}$ and $\theta$ (angle between $\vec{p}_{2}$ and $\vec{p}_{1}$ ) and $\epsilon_{3}$ and $\theta_{3}$ (angle of $\vec{p}_{3}$ with $\left.\overrightarrow{\mathrm{p}}_{1}+\overrightarrow{\mathrm{p}}_{2}\right)$. As in the 3D treatment ${ }^{9}$ the $\theta_{3}$ variable is changed to $\epsilon_{4}$; however, it is in this change that a fundamental difference with $3 \mathrm{D}$ arises. Figure 1 shows the geometry of a collision between opposite spins. From the figure one has $p_{4}{ }^{2}=p_{3}{ }^{2}+l^{2}-2 l p_{3} \cos \theta_{3}$, where $l=\left|\overrightarrow{\mathrm{p}}_{1}+\overrightarrow{\mathrm{p}}_{2}\right|$ so that

$$
d \theta_{3}=m d \epsilon_{4} / l p_{3} \sin \theta_{3} \text {. }
$$

If now we followed the 3D treatment, the denominator of (7) would be evaluated by placing all momenta on appropriate Fermi circles. However, the denominator in (7) would then become $p_{+} p_{\text {- }}$ $\times \sin \theta$, so that the integral over $\theta$ would diverge logarithmically at $\theta=0$ and $\pi$ (in $3 \mathrm{D}, \sin \theta$ in the solid angle eliminates any divergence). Obviously we must be more careful in 2D. By a bit of geometry we can show that

$$
\begin{array}{r}
\int_{0}^{2 \pi} d \theta \int_{0}^{\infty} d \epsilon_{3} \int_{0}^{\infty} d \epsilon_{4} \frac{\delta\left(\epsilon_{1}+\epsilon_{2}-\epsilon_{3}-\epsilon_{4}\right)}{l p_{3} \sin \theta_{3}}(\cdots) \\
\cong \frac{1}{2 m} \int_{0}^{2 \pi} d \theta \int_{\bar{\epsilon}-b}^{\bar{\epsilon}+b} d \epsilon_{3} \frac{(\cdots)}{\left[b^{2}-\left(\epsilon_{3}-\bar{\epsilon}\right)^{2}\right]^{1 / 2}},
\end{array}
$$

where $\bar{\epsilon}=\frac{1}{2}\left(\epsilon_{+}+\epsilon_{-}\right)$is the average Fermi energy and $b^{2}=\frac{1}{4}\left(\epsilon_{+}-\epsilon_{-}\right)^{2}+\epsilon_{+} \epsilon_{-} \sin ^{2} \theta$. (Note that we have set $\epsilon_{1}=\epsilon_{+}$and $\epsilon_{2}=\epsilon_{-}$but not $\epsilon_{3}=\epsilon_{+}$) In (8) the integration over $\epsilon_{4}$ gives unity whenever $\epsilon_{3}$ is

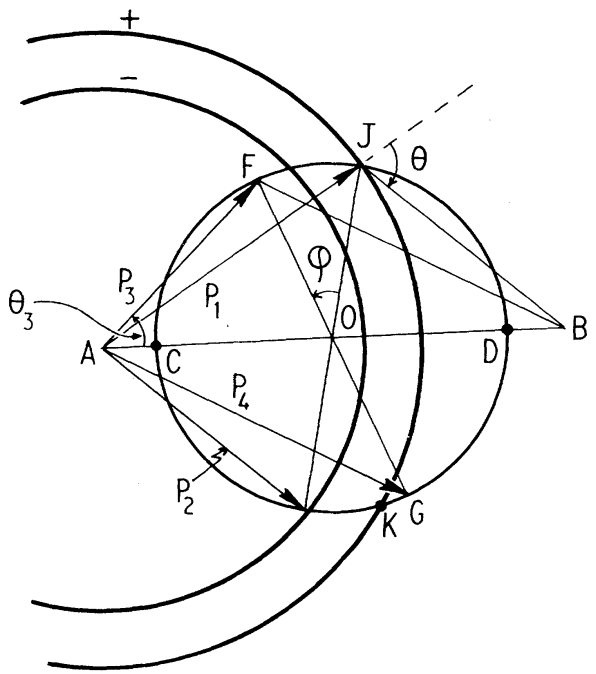

FIG. 1. Kinematics of a $(+,-)$ collision. The two Fermi circles are labeled + and -; $A B=\overrightarrow{\mathrm{p}}_{1}+\overrightarrow{\mathrm{p}}_{2}$. Energy and momentum conservation can be used to show that all $\overrightarrow{\mathrm{p}}_{i}$ lie on an "energy circle" (centered at 0 ). When $\overrightarrow{\mathrm{p}}_{3}$ and $\overrightarrow{\mathrm{p}}_{4}$ are on the Fermi circles, one sees that the scattering angle $\varphi$ has only two possible values, forward, with $\boldsymbol{F} \equiv J(\varphi=0)$, and backward with $G \equiv K(\varphi$ $\approx \pi$ for small polarization).

within the limits shown which occur at points C and $\mathrm{D}$ on the energy circle in Fig. 1. One sees that the $\epsilon_{3}$ density of states is integrably singular at these points. In the unpolarized case, where $\Delta<\theta<\pi-\Delta$ where $\Delta=k \boldsymbol{T} / \epsilon_{\mathrm{F}}$, the term $\left(\epsilon_{3}-\bar{\epsilon}\right)^{2}$, which is $\lesssim(k T)^{2}$ because of the Fermi distribution functions included in $(\cdots)$, can be dropped compared to $b^{2}=\epsilon_{\mathrm{F}}{ }^{2} \sin ^{2} \theta$ and the $\epsilon_{3}$ limits can be taken as 0 and $\infty$. For this region of $\theta$, Eq. (8) gives a result of order $T \ln T$. For $|\theta| \leqslant \Delta$ and $\pi-\Delta$ $\leqslant \theta \leqslant \pi+\Delta$ the $\epsilon_{3}$ factor cannot be dropped but Eq. (8) can be seen to give a contribution of order $T$ and this region of integ ration may be neglected relative to the other. Thus an effective "cutoff" at $\theta=\Delta$ is introduced.

From Fig. 1 one can see that, as long as $\theta$ is not within $\Delta$ of 0 or $\pi$, the scattering angle in $2 \mathrm{D}$ is limited to the forward direction $(\varphi=0)$ and to backward scattering $\left[\varphi=\varphi_{B}\left(p_{+}, p_{-}, \theta\right)\right.$, not quite equal to $\pi$ in a polarized system]. Thus the angles $\theta_{i}$ in $\Phi_{p \sigma}$ must be evaluated appropriate to forward and backward scattering. The contribution from forward scattering vanishes. (Such a scattering does not impede a spin current.) Also in a variational approach no scattering between like spins occurs. ${ }^{10}$ After carrying out all the integrations, ${ }^{9}$ we find

$$
q^{-1}=\frac{m^{3}(k T)^{2}}{8 \pi^{2} \hbar^{4}} \frac{\left(p_{+}{ }^{2}+p_{-}{ }^{2}\right)}{p_{+} p_{-}} \int_{\Delta}^{\pi-\Delta} d \theta \frac{\sin \theta w_{+-}\left(\theta, \varphi_{B}\right)}{p_{+}{ }^{2}+p_{-}{ }^{2}+2 p_{+} p_{-} \cos \theta} .
$$


We note that in the polarized case the angular factors (from the $\Phi_{i}$ ) have canceled out any divergence as $\theta \rightarrow 0, \pi$; however in the unpolarized case the integrand in (9) behaves as $(1-\cos \theta) / \sin \theta$ which diverges at $\pi$ and requires attention to the cutoff there. We examine Eq. (8) in several special cases:

(i) Unpolarized fluid $\left(p_{+}=p_{-}=p_{f}\right)$. The angular factor in Eq. (11) peaks up sufficiently sharply as $\theta \rightarrow \pi$ that it is accurate to leading order in temperature to evaluate $w_{+-}$at $\theta=\pi$ with $\varphi_{B}=\pi$ and take it out of the integral. Then Eqs. (6) and (11) yield

$$
D=\frac{8 \pi^{2} \hbar^{4}}{m^{3} w_{+-}(\pi, \pi)}\left(\frac{T_{f}}{T}\right)^{2} \frac{1}{\ln \left(T_{f} / T\right)} .
$$

As we have seen, the $\ln T$ behavior arises because the density of states behaves like $1 / \sin \theta$ until $\theta$ gets to within $\Delta$ of $\pi$.

(ii) Weakly polarized fluid $\left(k T \ll \epsilon_{+}-\epsilon_{-} \ll \bar{\epsilon}\right)$. In this case the angular factor in Eq. (9) still behaves like $1 / \sin \theta$ as $\theta \rightarrow \pi$; however, when $\theta$ approaches to within an angle $\sim\left(\epsilon_{+}-\epsilon_{-}\right) / \bar{\epsilon} \sim P \equiv\left(n_{+}\right.$ $\left.-n_{-}\right) / n$ it drops quickly to zero providing a different cutoff $(\gg \Delta)$ than in the unpolarized case. The result for $D$ is the same as Eq. (10) with $\ln \left(T_{f} / T\right)$ replaced by $\ln (1 / P)$. Physically this cutoff in angular factor is a result of the fact that backscattering $(\varphi=\pi)$ violates momentum conservation in a head-on collision $(\theta=\pi)$ when $\left|\vec{p}_{1}\right|=\left|\vec{p}_{3}\right|$ $=p_{+} \neq\left|\vec{p}_{2}\right|=\left|\vec{p}_{4}\right|=p_{.}$. P olarizations which satisfy the criteria of this section should be relatively easy to achieve by "brute force" fields and perhaps by optical pumping.

(iii) Highly polarized fluid $\left(k T \ll \epsilon_{-} \ll \epsilon_{+}\right)$. One finds from Eq. (9) that

$$
D=\frac{32 \pi^{2} \hbar^{4}}{m^{3}}\left(\frac{n_{-}}{n}\right)^{3 / 2}\left(\frac{T_{+}}{T}\right)^{2}\left[\int_{0}^{\pi} w_{+-}\left(\theta, \varphi_{B}\right) \sin \theta\right]^{-1}
$$

where $T_{+}=\epsilon_{+} / k$. Note that the logarithmic dependence is now absent and that $\varphi$ is actually closer to $2 \pi$ than $\pi$ for large polarization.

Equation (3) may be solved exactly to leading order in $T$ in 2D for arbitrary polarization. The reason this is possible is because we have been able to separate energy and angular integrations as shown in the paragraph between Eqs. (8) and (9). Once this is done, procedures similar to those in 3D (Ref. 11) may be followed. Furthermore, for the unpolarized case we are able to evaluate $D$ in terms of the Landau theory of Fermi liquids. ${ }^{9}$ We find

$$
D=\frac{\hbar}{\pi m *} \frac{\left(1+F_{0}{ }^{a}\right) C(\lambda)}{\left[\sum_{m}(-1)^{m} A_{m}{ }^{a}\right]^{2}}\left(\frac{T_{f}}{T}\right)^{2} \frac{1}{\ln \left(T_{f} / T\right)} .
$$

The $F_{m}{ }^{i}$ and $A_{m}{ }^{i}$ appearing in Eq. (12) are the 2D analogs of the 3D dimensionless Landau interaction and scattering amplitude parameters, respectively ( $i=s$, symmetric; $a$, antisymmetric). In 2D, functions of $\theta$ are expanded in terms of $\cos m \theta_{0}^{7}$ One can show that the $F_{m}$ and $A_{m}$ are related by $A_{m}{ }^{i}=F_{m}{ }^{i} /\left(1+c_{m} F_{m}{ }^{i}\right)$, where $c_{m}=1$ if $m$ $=0$ and $\frac{1}{2}$ otherwise. ${ }^{7}$ The factor $C(\lambda)$ is the Sykes and Brooker ${ }^{11}$ correction factor for relating the variational and exact diffusion constants; $\lambda$ is a parameter expressible in terms of ${A_{m}}^{s}$ and $A_{m}{ }^{a}$. Details will be given elsewhere.

The fact that an exact solution is possible is of interest since so few examples of solvable Boltzmann equations are available. However, the manner in which $D$ is expressible in terms of Landau parameters is perhaps of greater interest. The squared sum in the denominator of Eq. (12) is proportional to $w_{+-}(\pi, \pi)$. The Landau-theory scattering amplitude applies only to $\varphi=0$ (and by symmetry also to $\varphi=\pi)$. Any attempt to express scattering amplitudes at angles $\varphi$ other than 0 and $\pi$ (as must be done for 3D transport coefficients) involves interpolation and approximation. However, in 2D for weak or zero polarization only $\varphi=\pi$ is involved in $w_{+-}(\theta, \varphi)(\lambda$ also includes terms in $\varphi=0)$. Thus the static thermodynamic properties and transport coefficients can be expressed without approximation in terms of the Landau parameters. One might hope then that the measurement of the properties of a degenerate adsorbed ${ }^{3} \mathrm{He}$ system might lead to a more clearcut test of Landau's theory than is possible in bulk ${ }^{3} \mathrm{He}$ systems. The logarithmic temperature or polarization dependence of $D$ would represent a reliable indication that the system being examined was truly $2 \mathrm{D}$ and degenerate. The fact that a large number of Landau parameters seems to enter into Eq. (12) should present no difficulty. If the estimate given by Havens-Sacco and Widom ${ }^{7}$ is accurate, the sum over $A_{m}$ in (12) should be in powers of $\left(k_{f} a\right)^{2} / 8 \ln \left(k_{f} a\right) \approx 0.01$ for all coverages of interest, where $k_{f}=p_{f} / \hbar$ and $a$ is the scattering length. ${ }^{7}$ Thus only one or two Landau parameters need be kept in practice. We hope that experiments as suggested may be possible in the near future. The $2 \mathrm{D}{ }^{3} \mathrm{He}$ liquid reported in Ref. 4 may provide a suitable system for such experiments.

Useful discussions with M. T. Béal-Monod, R. A. Gyer, F. Laloë, A. J. Leggett, and C. Lhuillier are gratefully acknowledged. One of us (K.M.) wishes to thank the Nishina Memorial Foundation for support and the other (W.J.M.) is 
grateful to the Science Research Council for a Senior Postdoctoral Fellowship at Sussex.

(a) Permanent address: Department of Physics, Nagoya University Nagoya 464, Japan.

(b) Permanent address: Department of Physics and Astronomy, Hasbrouck Laboratory, University of Massachusetts, Amherst, Mass. 01003.

${ }^{1}$ For a review of properties of adsorbed ${ }^{3} \mathrm{He}$ see J. G. Dash, Films on Solid Surfaces (Academic, New York, 1975).

${ }^{2}$ J. R. Owens-Bradley, B. P. Cowan, M. G. Richards, and A. L. Thomson, Phys. Lett. 65A, 424 (1978); M. G. Richards, J. Phys. (Paris) Colloq. 39, C6-1342 (1978).

${ }^{3}$ R. C. Wayne and R. M. Cotts, Phys. Rev. 151, 264 (1966); B. Robertson, Phys. Rev. 151, 273 (1966).

${ }^{4}$ B. Bhattacharyya and F. M. Gasparini, Phys. Rev. Lett. $\underline{49}, 919$ (1982).

${ }^{5}$ We treat only longitudinal spin diffusion and neglect the Leggett-Rice effect [A. J. Leggett, J. Phys. C $\underline{12}$, 448 (1970)] or "spin rotation" effects [C. Lhuillier and F. Laloë, J. Phys. (Paris) 43, 197, 225 (1982)].

${ }^{6}$ The behavior $D^{-1} \sim T^{2} \ln T$ in an unpolarized $2 \mathrm{D}$ fluid was found previously in a numerical variational calcu- lation by H. Fu and C. Ebner, Phys. Rev. A 10, 338 (1974). C. Hodges, H. Smith, and J. W. Wilkins [Phys. Rev. B $\underline{4}, 302$ (1971)] found similar behavior in considering the electron scattering rate and resistivity of a metal having a cylindrical Fermi surface.

${ }^{7}$ Landau-theory treatments of the equilibrium properties of a 2D Fermi liquid have been given by R. Freedman, Phys. Rev. B 18 , 2482 (1978); S. M. Havens-Sacco and A. Widom, J. Low Temp. Phys. 40, 357 (1980). Also see P. Bloom, Phys. Rev. B 12, 124 (1975); L. Bruch, Physica (Utrecht) 94A, 586 (1978); M. B. Vetrovec and G. M. Carneiro, Phys. Rev. B 22, 1250 (1980).

${ }^{8}$ The validity of a Landau theory of a $2 \mathrm{D}$ Fermi system has been questioned by M. Gabay and M. T. BéalMonod, Phys. Rev. B 18, 5033 (1978). We have not resolved this question and simply assume the validity of Landau theory in the present paper.

${ }^{9}$ A. A. Abrikosov and I. M. Khalatnikov, Rep. Prog. Phys. 22, 329 (1959); D. Hone, Phys. Rev. 21, 669 (1961); G. Baym and C. J. Pethick, in The Physics of Solid and Liquid Helium, edited by K. H. Benneman and J. B. Ketterson (Wiley, New York, 1977), Pt. II. ${ }^{10}$ V. J. Emery, Phys. Rev. 133, A661 (1964). ${ }^{11} \mathrm{~J}$. Sykes and G. A. Brooker, Ann. Phys. (N.Y.) $\underline{56}, 1$ (1970).

\title{
Scaling Treatment of Critical and "Chaotic" Dynamics of the Dilute Heisenberg Chain
}

\author{
R. B. Stinchcombe \\ Department of Theoretical Physics, Oxford University, Oxford OX1 3NP, United Kingdom
}

(Received 13 October 1982)

\begin{abstract}
A lattice rescaling method is applied to the equations of motion of the dilute Heisenberg chain and leads via a probabilistic integral equation to an iterative map for the characteristic frequency $\beta$ and concentration $p$. Dilution induces a crossover in the $\beta$ scaling from "chaotic" (ergodic and mixing) behavior, corresponding to the sampling of the pure band, to periodic orbits corresponding to isolated cluster response. A dynamic scaling form is obtained for the critical dynamics by fixed-point analysis.
\end{abstract}

PACS numbers: $64.60 . \mathrm{Fr}, 64.60 . \mathrm{Ht}, 75.30 . \mathrm{Ds}, 75.40 . \mathrm{Fa}$

Much recent progress has been made in understanding static critical properties of disordered systems by the use of lattice rescaling methods. ${ }^{1}$ Up to now, however, there has been no satisfactory development of such methods for the dynamic properties, partly because of some constructional and interpretive difficulties in lattice rescaling methods for the dynamics of the pure case $e^{2}$ and partly because of insufficient care with the disorder. These difficulties are overcome in this Letter. I will treat the particular case of the diluted Heisenberg chain at absolute zero.

I give here the first lattice-rescaling treatment transforming the distribution function of the $d y$ - namic variable. I extract an equation for the scaling of the characteristic frequency which is able to deal with the critical dynamics and to treat both band (extended) and localized (cluster) response. The scaling equation is an iterative map $^{3-5}$ with control variable ranging, as the concentration variable scales, from values yielding "chaotic" behavior associated with band response to values yielding a hierarchy of bifurcated stable orbits corresponding to isolated cluster dynamics.

If one is not interested in the full crossover, the low-frequency (critical) dynamics can be obtained simply by linearizing about the doubly unstable zero-frequency percolation fixed point of 